Session $12-23$

\title{
Ultra Low Cycle Fatigue of Axisymmetric Freestanding Nanoscale Gold Films
}

\author{
Khawar Abbas, Zayd C. Leseman \\ Department of Mechanical Engineering \\ University of New Mexico \\ Albuquerque , NM 87131 \\ Thomas J. Mackin \\ Department of Mechanical Engineering \\ California Polytechnic State University \\ San Luis Obispo, CA 93407
}

\begin{abstract}
Experimental measurements of axisymmetric nano-crystalline gold films of nano-thickness after they have been plastically deformed by a spherical indenter are presented in this paper. Freestanding membranes of gold are centrally deflected using a spherical indenter attached to a MEMS load cell. Films fabricated for these experiments were $100 \mathrm{~nm}$ thick and $500 \mu \mathrm{m}$ in diameter. We observed that the plastically deformed thin films recover completely in time at room temperature. One particular film was loaded 4 consecutive times, with the fourth loading leading to its fracture after full recover from the three prior loadings. We termed this behavior as the "Ultra Low cycle fatigue".
\end{abstract}

\section{Introduction}

Micro Electro Mechanical Systems (MEMS) and its offshoot Nano Electro Mechanical Systems (NEMS) have been very popular all over the world lately. At the heart of it, is the support from a very mature semiconductor technology. This bolstered interest and the presence of a well developed technology base has resulted in a very rapid growing commercial market for the MEMS and NEMS devices. Haque et. al. ${ }^{1}$ lists some of these devices. Many of the commonly used MEMS/NEMS devices have metallic thin films as mechanical structures. The elastic and plastic properties of these thin films are differ significantly from those of the bulk material ${ }^{2,3,4}$. At these scales the volume fraction of material defects such as: grain boundaries, dislocations and interstitials become quite significant and become a chief contributor the physical and mechanical material properties of the thin films. Some of the popular thin film materials used in MEMS/NEMS are Aluminum (Al), Copper $(\mathrm{Cu})$, Nickel $(\mathrm{Ni})$ and Gold $(\mathrm{Au})$. However Gold $(\mathrm{Au})$ is usually preferred due to its high electrical conductivity, chemical inertness and resistance to oxidation.

Mechanical properties of thin films have been studied by many researchers in the past. Vinci et al. ${ }^{4}$ developed and described several specialized techniques to determine the mechanical properties and stress strain states of both free standing and films bonded to substrate. He described nanoindentation as a popular and effective way of determining the elastic and plastic properties as well as hardness of 
free standing thin films. Detailed theoretical and experimental research of the atomistic and molecular mechanism of adhesion, contact formation, nanoindentation, and fracture that occurs when a Ni diamond shaped nanoindenter interacts with the Au surface is provided by Landman et. al. ${ }^{5}$. Measurements of Young's modulus on free standing thin films and observed the relaxation of thin films at room temperature with frequency dependence were made by Kalkman et al. ${ }^{3}$. They attribute this anelastic behavior to the grain boundary slipping. Haque et al. ${ }^{1}$ studied the relaxation of freestanding nanocrystalline $\mathrm{Au}$ films at room temperature and used an analytical model based on a spring and a dashpot to predict instantaneous Young's modulus. They also demonstrate the effect of size in nanoscale solids by comparing the relaxation time at room temperature with that of bulk solids.

In addition to relaxing under the stress, ductile metals at the nano-scale also exhibit property of coalescence and adhesion. Alcanter et al. ${ }^{6}$ observed that when surfaces composed of nano scale asperities in $\mathrm{Au}$ and $\mathrm{Pt}$ films are pressed together they sinter or cold weld at nano-scale to form a continuous bulk film. Kuczynski ${ }^{7}$ also displayed experimentally the self diffusion of $\mathrm{Cu}$ and $\mathrm{Ag}$ particles to the flat surface of the same metal at temperatures below their melting points and measured the coefficient of self diffusion and rate of interface growth between the round particles and a metallic block. Strouse et al also reports that the Au particles at nano-distances with each other suspended in organic fluids coalesce to form a continuous material having single monolithic interface $^{6}$. Recently Rajgopalan et. al. ${ }^{8}$ observed the complete healing and recovery of $\mathrm{Al}$ and $\mathrm{Au}$ films when annealed subsequent to the plastic deformation.

Very few studies have been conducted so far that explain the failure mechanism of free standing thin films due to fatigue. Hadboletz et al. ${ }^{9,10}$ studied the crack growth in free standing $\mathrm{Cu}$ foils of different thicknesses when subjected to high cycle bending fatigue. Different studies indicate the fatigue behavior of thin films is dependent upon their thickness. Studies on thin films exhibiting ultra low cycle fatigue ( $<10$ cycles) are not available.

In short, the mechanical and physical properties of the thin films differ substantially from those of the bulk material. There are a wide range of studies available that describe the nanoindentation method of testing, behavior of the thin films under load and their ability to relax at room temperature when put under a stress. However there are not many recorded experimental observations that show the property of ductile metals in general and $\mathrm{Au}$ in particular to coalesce at nano-scale and recover by itself over finite time duration at room temperature and subsequently fracture due to ultra low cycle fatigue. Our novel experiment, described subsequently, is an attempt to observe and study this behavior.

\section{Experimental Setup}

The experimental setup used for these experiments is similar to that described by Leseman et al. ${ }^{11}$. The experimental setup is composed of four components: a load cell, a freestanding axisymmetric thin film, a system of high precision linear and rotational stages, and two microscopes. The load cell is fabricated by microfabrication techniques. The wafer is cleaved and a portion of the device (the testing tip) is allowed to hang over the edge of the Si wafer. A novel technique for the calibration of load cells is employed ${ }^{12}$, wherein calibrated weight are hung from the load cell and their 
corresponding displacements are recorded. Finally, a sapphire sphere of known mass is epoxied to the load cell, see Figure 1.

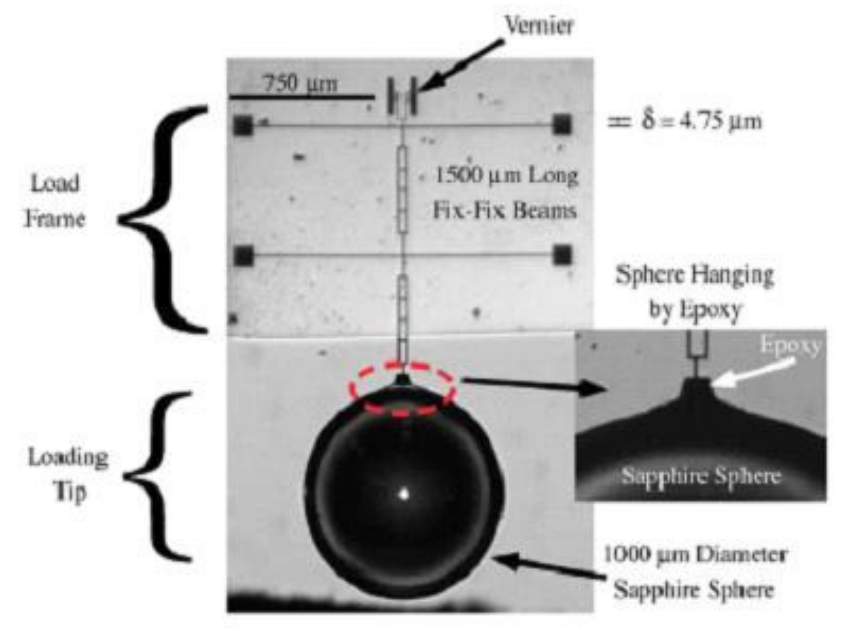

Figure 1: A $1000 \mu \mathrm{m}$ ball lens epoxied to the load cell

The freestanding film is also fabricated using microfabrication techniques. An array of circles of varying diameters ( $1 \mu \mathrm{m}$ to $2 \mathrm{~mm}$ ) was transferred onto the wafer using standard photolithographic techniques. Each array of circles is contained within a larger circle measuring $2.75 \mathrm{~mm}$ in diameter. The smaller circles define the pattern of the freestanding membranes and the larger circle is for ease of handling in the TEM*. XRD Analysis of the films showed a compressive $10 \mathrm{MPa}$ residual stress contained in the thin film. This result was also confirmed by the residual stress measurement, which utilized Stoney's Formula. TEM inspection allowed us to determine the average grain size which is $57 \mathrm{~nm}$.

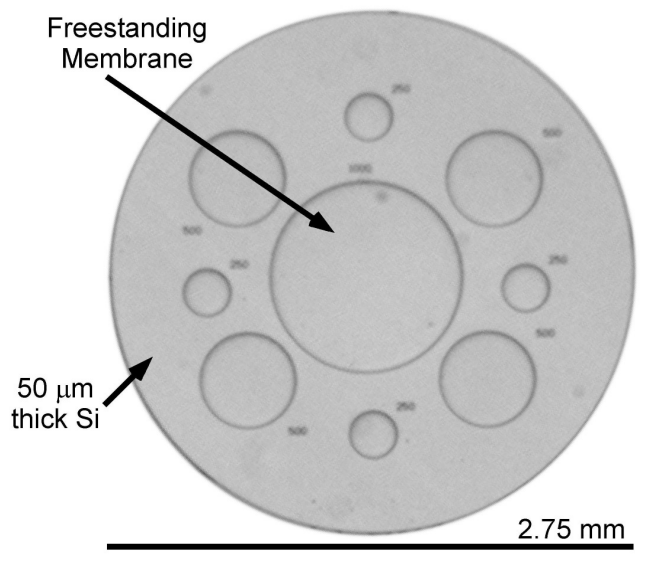

Figure 2: Large circular array containing several free standing gold films. The array fits TEM sample perfectly enabling pre and post-test inspections 
MEMS load cell is positioned above the freestanding thin film by high precision linear and rotational stages. The piezoelectric stage is of particular importance, it allows incremental motion $(50 \mathrm{~nm})$ of the load cell into the fixture freestanding thin films, see Figure 3.

In-situ observation of the experiment is made by two microscopes. First, an interferometric microscope is used to observe the thin film. By constructive and destructive interference of a specific wavelength of light $(548 \mathrm{~nm})$ the displacements into and out the page at a resolution of 137 $\mathrm{nm}$ can easily discerned. The second microscope is used to monitor the vernier located atop the load cell. The vernier allows us to measure the displacement at the centerline of the load cell with a resolution of $500 \mathrm{~nm}$.

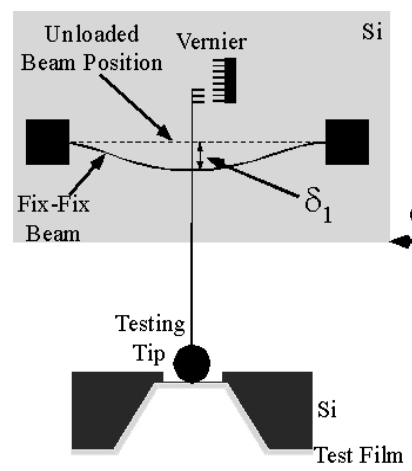

(A)

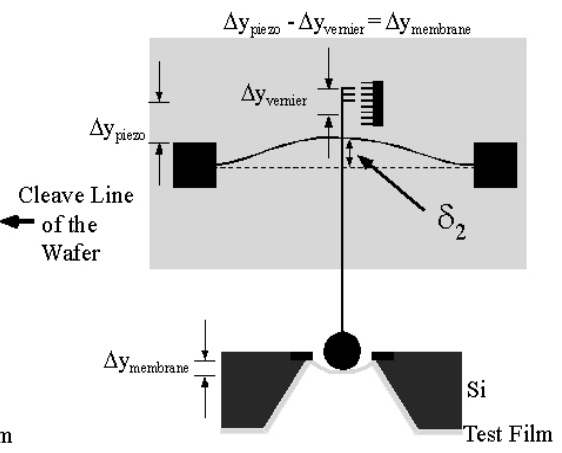

(B)

Figure 3: Schematic representation of the micromechanical membrane testing system. a testing tip is co-fabricated with the fixed fixed beam on a silicon substrate. A vernier on the back side of the beam measures the beam displacement during testing

The difference between the piezoelectric crystals displacement and the load cell's vernier measurement gives the displacement of the center point of the freestanding thin. As a cross check we use the displacement profile of the membrane as seen by the interferometric microscope. A schematic of the full apparatus is shown in Figure 4.

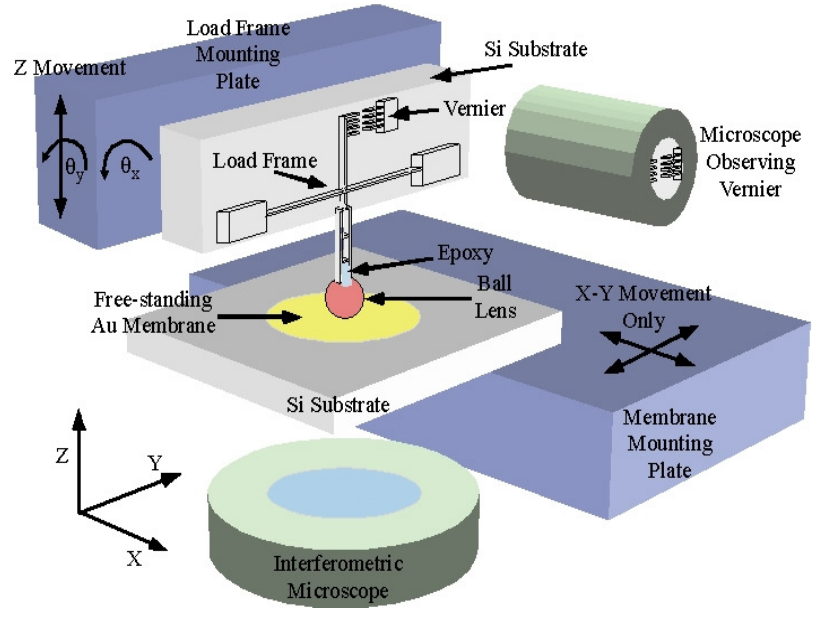

Figure 4: Schematic of the experimental apparatus 


\section{Experiment}

Loading history of a single membrane is described is here. Similar behavior was also observed in other experiments with membranes of similar dimensions. This observation is borne out as the result of other similar experiments.

Experiments were carried out in a laboratory setting (not in a cleanroom), temperature and relative humidity were $20{ }^{\circ} \mathrm{C}$ and $\sim 50 \%$, respectively. The experimental apparatus of Figure 3 was mounted on a vibration isolation table.

Loading cycles of the membrane are subsequently described. Initially, the membrane is loaded to some maximum value, Figure 4a. Unloading ensues, but does not terminate at zero load. During loading, the membrane has adhered to the sapphire sphere, Figure $5 \mathrm{~b}-5 \mathrm{c}$. Thus zero load, has a positive finite displacement, see Figure 5. After further movement of the sphere away from the centerline of freestanding thin film, the film suddenly releases from the film and recovery begins.
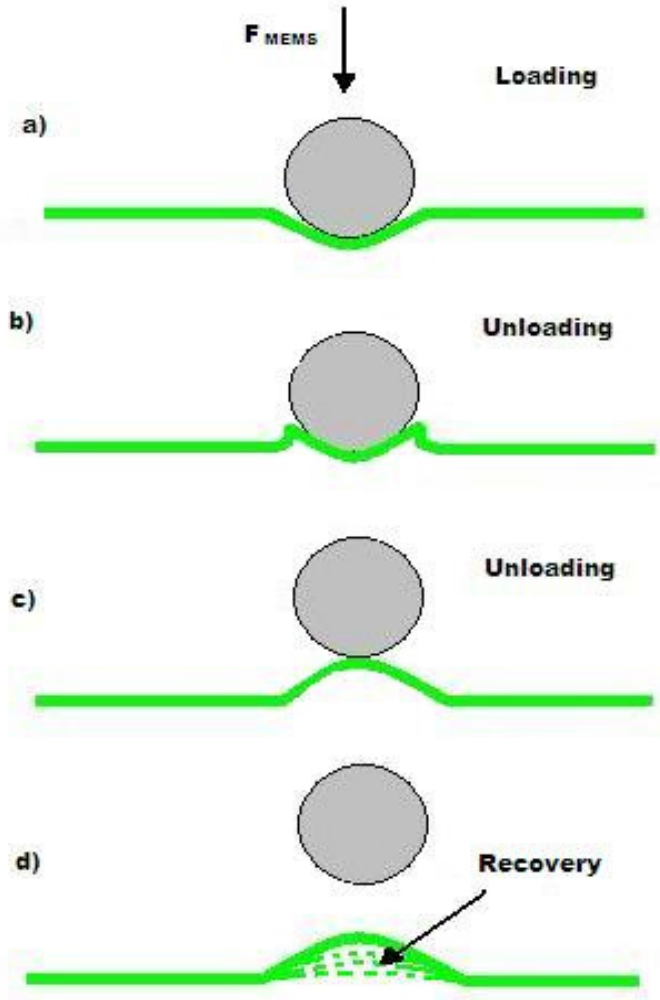

Figure 5: Condition of the film while load is removed after the plastic deformation

\section{Results}


The gold membranes was loaded to the max load of $691 \mu \mathrm{N}$ and $761 \mu \mathrm{N}$ and the complete 'healing' was observed to occur in time varying between 6 seconds to 36 minutes! With time required to heal increasing with each cycle. Figure 6 shows the state of the film at various time intervals. Figures 7 show the trend of decreasing deformation diameter while healing when the film is loaded up to $691 \mu \mathrm{N}$ and later load is removed. The error bars in figures 7 are due to graphical method of diameter measurement.

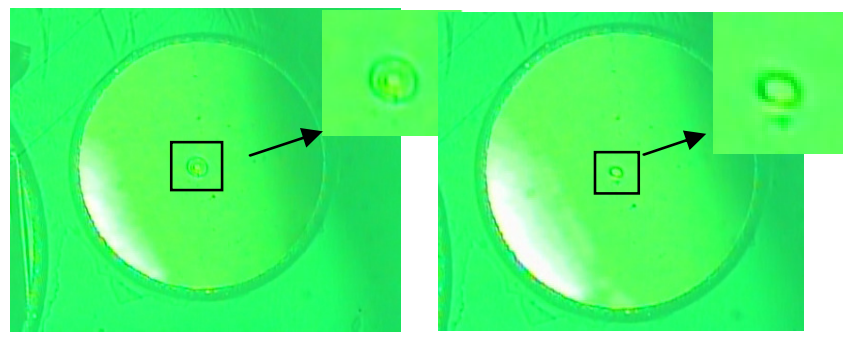

$\mathrm{T}=0 \mathrm{~min}$

$\mathrm{T}=1: 30 \mathrm{~min}$
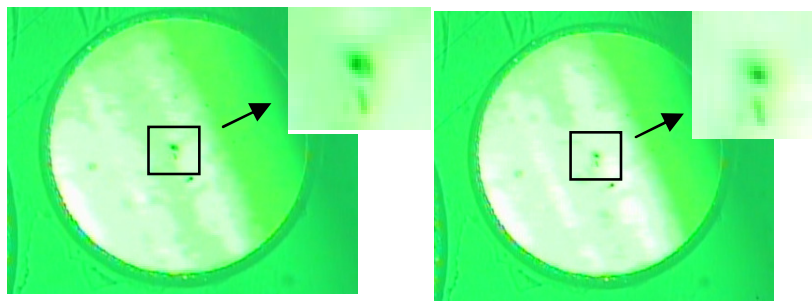

$\mathrm{T}=3 \mathrm{~min}$

$\mathrm{T}=4 \mathrm{~min}$

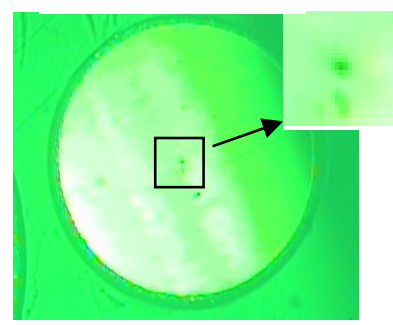

$\mathrm{T}=5 \mathrm{~min}$

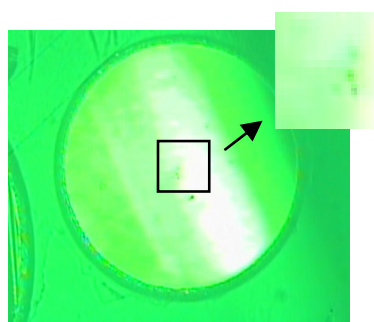

$\mathrm{T}=6 \mathrm{~min}$

Figure 6: Decrease in the diameter of deformation caused by the indentation, which gradually disappears completely 


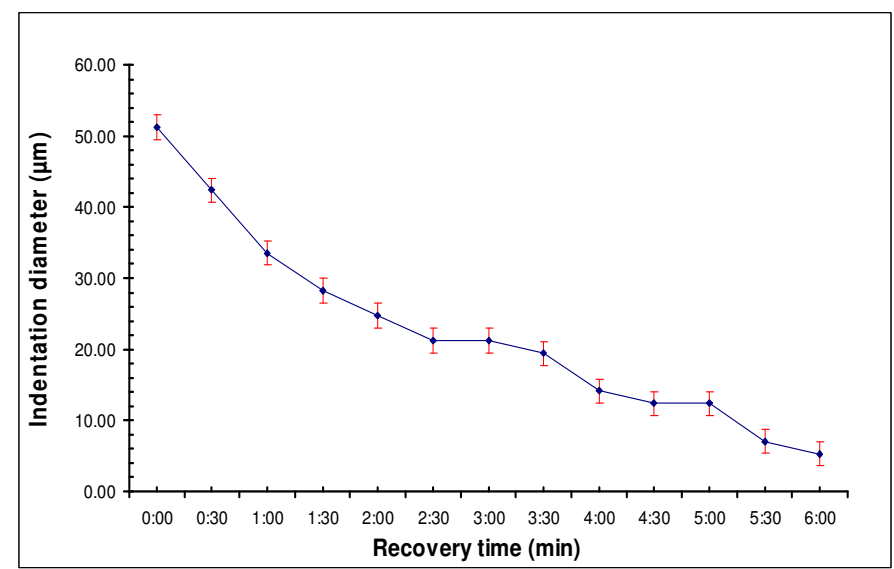

Figure 7: Trend of decreasing indentation diameter vs time when the film was loaded to $761 \mu \mathrm{N}$ and later unloaded for the first time. The complete recovery took 6 minutes.

Figure 7 shows the displacement of the film and its decreasing trend with the passage of time. This data was collected from images obtained with the interferometric microscope. The distance between the fringes caused in the film by the indentation is $\lambda / 2$, where $\lambda$ is the wave length of the light used in the interferometer. The value for which was $548 \mathrm{~nm}$. Therefore vertical height between corresponding fringes (similarly colored) is $274 \mathrm{~nm}$, and vertical height between the dark spot and a light spot would then be $137 \mathrm{~nm}$. Images were captured at regular interval while the film was healing. These images were edited graphically and circles drawn to fit each fringe pattern graphically. These circles were subsequently measured and corrected for difference of scale giving the radius of the fringe pattern at each interval. Note that the overall deformed shape varies only slightly at $\mathrm{t}=0$. The heights and diameters of the diameters of the deformed shape are nearly the same, yet the time for recovery differed by $\sim 600 \%$.

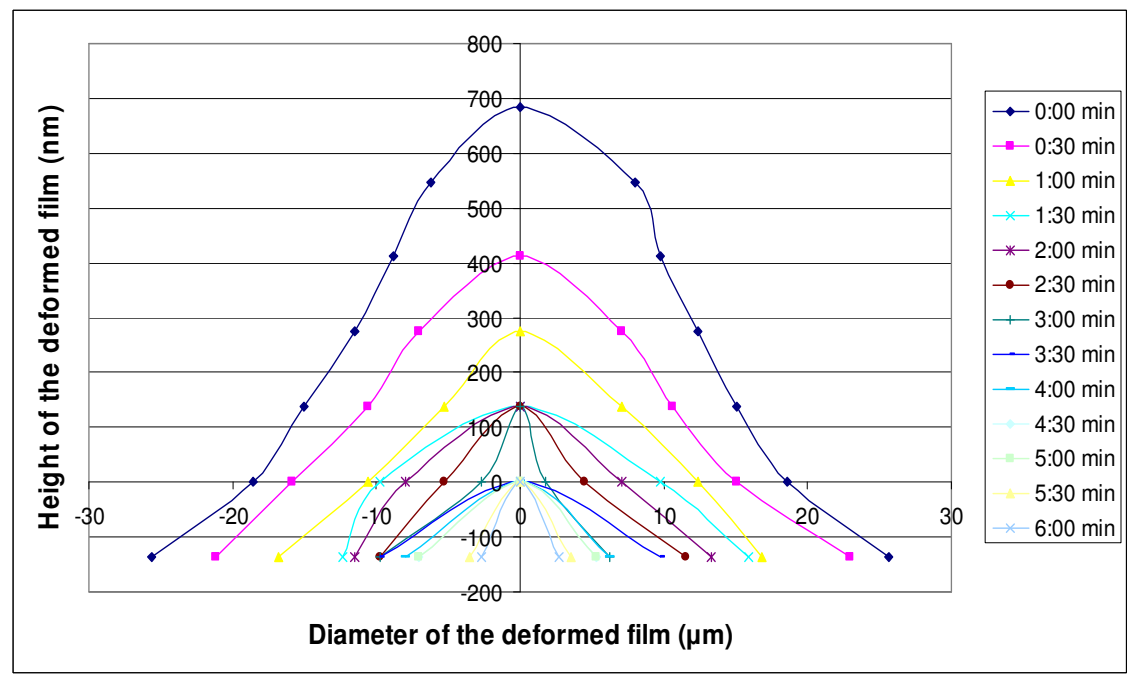

Figure 7: Gradual decrease in the indentation depth and the diameter with time when the film was loaded up to $761 \mu \mathrm{m}$ and later unloaded for the first time. 
Additionally the membrane eventually fractured on the fourth cycle indicating the presence of low cycle fatigue. TEM analysis of the fractured film also shows intra-granular and transgranular fracture that that fit the low cycle fatigue description.

\section{Conclusion}

There is a definitive evidence of the "self-healing" nature of freestanding thin Au films at room temperature. It demonstrates that the metals have the property to recover to a certain degree and repair the damage caused by the plastic deformations. The fracture of the film in 4 cycles also indicates the failure due to ultra low cycle fatigue. However, in order to explain the mechanism of self healing at the granular level and form a mathematical explanation further investigations are required.

\section{References}

1. M. A. Haque and B. A. Samuel, 2006, "Room Temperature relaxation of free standing nanocrystaline gold film," J. micromech. Microeng, pp. 929-934.

2. H. Huang and F. Spaepen, 2000, "Tensile testing of free-standing Cu, Ag and Al thin films and Ag/Cu multilayers," Acta Materialia, pp. 3261-3269.

3. A. J. Kalkman, A. H. Verbruggen, and C. A. M. Janssen, 2001, "Young's modulus measurement and grain boundary sliding in free standing thin metal films," American Institute of Physics, vol. 78, pp. 2673-2675.

4. R. P. Vinci, J. J. Vlassak, and Annu, 1996, "Mechanical Behavior of thin films," Rev. Mater. Sci, pp. 431-462.

5. U. Landman, W. D. Luedtke, N. A. Burnham, and R. J. Colton, 1990, "Atomistic Mechanism and dynamic adhesion, Nanoindentation and fracture," Science, New series, vol. 248, pp. 454-461.

6. N. A. Alcantar, C'. Park, J.-M. Pan, and J. N. Israelachvili, 2002, "Adhesion and coalescence of ductile metal surfaces and nano particles," Acta materialia, pp. 31-47.

7. G. C. Kuczynski, "Self diffusion in sintering of metallic particles, 1949, " AIME, pp. 169-178.

8. [8] J. Rajgopalan, J. H. Han, and M. T. Saif, 2006, "Plastic deformation recovery in freestanding nanocrystalinr Aluminum and gold thin films," Science, vol. 315, pp. 1831-1834.

9. A. Hadrboletz, B. Weiss, and G. Khatib, 2001, "Fatigue and fracture properties of thin metalic foil," International Journal of fracture, pp. 68-89.

10. A. Hadrboletz, B. Weiss, G. Khatibi, and Sticler, 1999, "Fatigue crack growth behaviour of thin metallic " Fatigue 99, Engineering Materials advisory services Ltd., UK, pp. 1865-1870.

11. Z. C. Leseman and T. J. Mackin, 2007, "Indentation testing of axissymmetric freestanding nanofilms using a MEMS load cell," to appear.

12. Z. C. Leseman and T. J. Mackin, 2007"A calibration procedure for custom-fabricated MEMS based load cells," Journal of Microelectromechanical Systems.

13. F. Larmer and A. Schlip, 1992, "Method for anisotropically etching silicon," USA.

14. Z. C. Leseman, in Mechanical Engineering. vol. PhD., 2006, Urbana Champaign: University of Illinois.

\section{KHAWAR ABBAS}

Khawar Abbas is currently a Graduate student of Mechanical Engineering Department at the University of New Mexico. He is currently working towards his Masters Degree in Mechanical Engineering.

\section{ZAYD C. LESEMAN}

Proceedings of the 2008 ASEE Gulf-Southwest Annual Conference

The University of New Mexico - Albuquerque

Copyright (C) 2008, American Society for Engineering Education 
Dr. Leseman currently serves as an Assistant Professor in Mechanical Engineering Department at the University of New Mexico. His research encompasses the design, fabrication, and analysis of novel MEMS/NEMS devices and experiments in order to study the surface, mechanical, and electrical properties of materials at the nanoscale. Additionally, he creates bioMEMS devices to perform studies in single-cell mechanics and nano/microfluidics.

\section{THOMAS J. MACKIN}

Dr. Mackin currently serves as Professor and Chair of the Mechanical Engineering Department at California Polytechnic State University. His research Interests are Mechanics of Materials, MEMS, NEMS, microfabrication, microelectronic packaging, non-destructive testing, thermoelastic stress analysis, failure analysis, science and technology policy, critical infrastructure protection 